\title{
Conformal Invariants for Determinants of Laplacians on Riemann Surfaces
}

\author{
William I. Weisberger \\ Institute for Theoretical Physics, State University of New York at Stony Brook, Stony Brook, \\ New York 11794-3840, USA
}

\begin{abstract}
For a Riemann surface with smooth boundaries, conformal (Weyl) invariant quantities proportional to the determinant of the scalar Laplacian operator are constructed both for Dirichlet and Neumann boundary conditions. The determinants are defined by zeta function regularization. The other quantities in the invariants are determined from metric properties of the surface. As applications explicit representations for the determinants on the flat disk and the flat annulus are derived.
\end{abstract}

\section{Introduction}

It is ancient lore that one can find the inverses of Laplace operators (Green's functions) on Riemann surfaces by conformal mappings from geometries where the potential theory problem is soluble directly. In this article I show that an analogous result applies to the calculation of determinants of Laplacians. By studying the variation under Weyl transformations of determinants of the scalar Laplacian on Riemann surfaces with smooth boundaries one can construct quantities proportional to the determinants which are invariant under Weyl transformations as well as reparameterization of the surface. ${ }^{1}$ The techniques used in the derivation are adapted from those used to study Weyl invariance and fix the critical dimension in Polyakov's path-integral formulation of string theory $[1,2,3]$.

To show the utility of these invariants, they are used to obtain explicit representations of the determinants of the scalar Laplacian for both Dirichlet and Neumann boundary conditions on the flat disk and the flat annulus. Zeta function regularization is used to define the formally divergent expressions for the determinants [4].

To write the invariants on a Riemann surface, $M$, with smooth boundaries, $\partial M$, we use the following notation. The scalar Laplacian is

$$
\Delta=-(1 / \sqrt{g}) \partial_{a}\left(\sqrt{g} g^{a b} \partial_{b}\right)
$$

1 The method is also applicable to Laplace operators acting on higher rank tensors 
Subscripts $D$ and $N$ denote quantities for Dirichlet (eigenfunctions of $\Delta$ vanishing on $\partial M$ ) and Neumann (normal derivatives of eigenfunctions vanishing on $\partial M$ ) boundary conditions respectively.

$$
\begin{aligned}
& A=\int_{M} d \mu \text { is the area of } M . \\
& P=\int_{\partial M} d s \text { is the perimeter of } \partial M . \\
& K \text { is the Gaussian curvature on } M . \\
& \kappa_{g} \text { is the geodesic curvature on } \partial M .
\end{aligned}
$$

On $M$ with a family of smooth metric tensors $\left\{g_{a b}\right\}$, a conformal gauge $\hat{g}_{a b}$ is defined by writing

$$
g_{a b} \sim e^{2 \phi} \hat{g}_{a b}
$$

where equivalence means equality up to a reparameterization. For a given gauge we define

$$
L\left(\phi ; \hat{g}_{a b}\right)=\int_{M} d \hat{\mu} \hat{K} \phi+\int_{\partial M} d \hat{s} \hat{\kappa}_{g} \phi+(1 / 2) \int_{M} d \hat{\mu} \hat{g}^{a b}\left(\partial_{a} \phi\right)\left(\partial_{b} \phi\right) .
$$

If $\hat{g}_{a b}$ is an admissible global gauge choice on $M$, the invariants are ${ }^{2}$

$$
I_{D}=\left(\operatorname{det}_{D} \Delta\right) \exp \left[L /(6 \pi)+(1 / 4 \pi) \int_{\partial M} \kappa_{g} d s\right]
$$

and

$$
I_{N}=\left(\operatorname{det}_{N}^{\prime} \Delta\right)(1 / A) \exp \left[L /(6 \pi)-(1 / 4 \pi) \int_{\partial M} \kappa_{g} d s\right] .
$$

The prime in the Neumann determinant denotes that the zero mode is omitted.

\section{Derivation}

If $\left\{\lambda_{i}\right\}$ denotes the eigenvalues of $\Delta$, the determinants can be defined by zeta function regularization as

$$
\operatorname{det}^{\prime} \Delta=\exp \sum_{i}^{\prime} \ln \lambda_{i}=\exp -\left.\frac{d}{d s} \zeta(s)\right|_{s=0},
$$

where

$$
\zeta(s)=\sum_{i}^{\prime} \lambda_{i}^{-s}
$$

We will evaluate the variation in $\operatorname{det}^{\prime} \Delta$ under an infinitesimal Weyl transformation

which induces

$$
g_{a b} \rightarrow(1+2 \delta \phi) g_{a b}
$$

$$
\Delta \rightarrow(1-2 \delta \phi) \Delta
$$

2 For a compact surface (no boundaries) we have the invariant

$$
I=\left(\operatorname{det}^{\prime} \Delta\right)(1 / A) \exp [L /(6 \pi)]
$$


The corresponding variation in an eigenvalue is

$$
\delta \lambda_{i}=\left\langle\psi_{i}|\delta \Delta| \psi_{i}\right\rangle=-2 \lambda_{i}\left\langle\psi_{i}|\delta \phi| \psi_{i}\right\rangle,
$$

where $\psi_{i}$ is the eigenfunction corresponding to $\lambda_{i}$. Also

$$
\delta \zeta(s)=-s \sum_{i}^{\prime} \delta \lambda_{i} / \lambda_{i}^{s+1}
$$

and

$$
\begin{aligned}
\delta\left(\ln \operatorname{det}^{\prime} \Delta\right) & =-2 \lim _{s \rightarrow 0} \sum_{i}^{\prime}\left\langle\psi_{i}|\delta \phi| \psi_{i}\right\rangle \lambda_{i}^{-s} \\
& =\lim _{s \rightarrow 0}[-2 / \Gamma(s)] \int_{0}^{\infty} d t t^{s-1} \sum_{i}^{\prime}\left\langle\psi_{i}|\delta \phi| \psi_{i}\right\rangle \exp \left(-t \lambda_{i}\right) .
\end{aligned}
$$

The singularity of the integral at $s=0$ which cancels the zero of $1 / \Gamma(s)$ arises from the small $t$ region. Therefore, we can split the integration range into the intervals $[0,1]$ and $[1, \infty]$ and discard the integration over the latter. This leads to the representation

$$
\begin{aligned}
\delta\left(\ln \operatorname{det}^{\prime} \Delta\right)= & 2 \sum_{\lambda_{i}=0}\left\langle\psi_{i}|\delta \phi| \psi_{i}\right\rangle \\
& -\lim _{s \rightarrow 0}[2 / \Gamma(s)] \int_{0}^{1} d t t^{s-1} \int_{M} d \mu \delta \phi G(\vec{r} \mid \vec{r}, t),
\end{aligned}
$$

where $G$ is the heat kernel which admits the representation

$$
G\left(\vec{r} \mid \vec{r}^{\prime}, t\right)=\sum_{i} \psi_{i}(\vec{r}) \psi_{i}^{\dagger}\left(\vec{r}^{\prime}\right) \exp \left(-t \lambda_{i}\right)
$$

For Dirichlet boundary conditions there is no zero mode. For Neumann boundary conditions, there is a single zero mode with a constant eigenvector which can be taken to be

Hence, in this case

$$
\psi_{0}=1 / \sqrt{A}
$$

$$
2\left\langle\psi_{0}|\delta \phi| \psi_{0}\right\rangle=\delta(\ln A) .
$$

To get the required singular terms from the $t$ integral we use the small $t$ expansion of the heat kernel which gives $[2,5,6]$

$$
\begin{aligned}
\int d \mu \delta \phi G_{\left(\begin{array}{c}
D \\
N
\end{array}\right)}(\vec{r} \mid \vec{r}, t)= & 1 /(8 \pi t) \delta A \mp 1 /\left[8(\pi t)^{1 / 2}\right] \delta P \pm 1 /(8 \pi) \delta \int_{\partial M} d s \kappa_{g} \\
& +1 /(12 \pi) \delta L+0\left(t^{1 / 2}\right),
\end{aligned}
$$

where

$$
\delta L=\int_{M} d \mu K \delta \phi+\int_{\partial M} d s \kappa_{g} \delta \phi .
$$

Only the $t$-independent terms give rise to the required $(1 / s)$ singularity after integration. The variation $\delta \phi$ is reparameterization and gauge invariant. If we choose a coordinate system and a gauge $\hat{g}_{a b}$ so that

$$
g_{a b}=e^{2 \phi} \hat{g}_{a b}
$$


the quantities appearing in $\delta L$ are related to those for the metric $\hat{g}_{a b}$ by

$$
\begin{aligned}
K & =e^{-2 \phi}[\hat{K}+\hat{\Delta} \phi], \\
\kappa_{g} & =e^{-\phi}\left[\hat{\kappa}_{g}-(\hat{n} \cdot \partial) \phi\right], \\
d \mu & =e^{2 \phi} d \hat{\mu}, d s=e^{\phi} d \hat{s} .
\end{aligned}
$$

$n^{a}$ is the inward pointing unit normal vector to the boundary. Then we can write

$$
\delta L\left(\phi ; \hat{g}_{a b}\right)=\int_{M} d \hat{\mu} \hat{K} \delta \phi+\int_{\partial M} d \hat{s} \hat{\kappa}_{g} \delta \phi+\int_{M} d \hat{\mu}(\hat{\Delta} \phi) \delta \phi-\int_{\partial M} d \hat{s}(\hat{n} \cdot \partial \phi) \delta \phi .
$$

If $\hat{g}_{a b}$ defines a global gauge choice, we can integrate the third term on the right by parts, and the boundary term will cancel the last term. However, for a local gauge choice which requires dividing the surface into coordinate patches, integrating by parts gives nonvanishing contributions from integrals over the internal boundaries between different patches. Assuming that a globally admissible gauge choice has been made, Eq. (18) can be integrated to obtain [2]

$$
L\left(\phi, \hat{g}_{a b}\right)=\int_{M} d \hat{\mu} \hat{K} \phi+\int_{\partial M} d \hat{s} \hat{\kappa}_{g} \phi+(1 / 2) \int_{M} d \hat{\mu} \hat{g}^{a b}\left(\partial_{a} \phi\right)\left(\partial_{b} \phi\right) .
$$

There is a constant of integration in the definition of $L$ which depends on our choice of $\hat{g}_{a b}$. Suppose $\phi \rightarrow \phi^{\prime}$ under a change of gauge $\hat{g}_{a b} \rightarrow \tilde{g}_{a b}$, where

$$
\hat{g}_{a b} \sim e^{2 \sigma} \tilde{g}_{a b}
$$

Then

$$
L\left(\phi, \hat{g}_{a b}\right)=L\left(\phi^{\prime}, \tilde{g}_{a b}\right)-L\left(\sigma, \tilde{g}_{a b}\right)
$$

Putting everything together we have

$$
\delta\left(\ln \operatorname{det}_{\left(\begin{array}{l}
D \\
N
\end{array}\right)}^{\prime} \Delta\right)=1 /(6 \pi) \delta L \mp 1 /(4 \pi) \delta \int d s \kappa_{g}+\left\{\begin{array}{c}
0 \\
\delta \ln A
\end{array}\right\} .
$$

Integrating gives the reparameterization Weyl invariants of Eqs. $(4,5)$.

\section{Applications}

A. Flat Disk of Radius $r$. For Dirichlet and Neumann boundary conditions the eigenvalues of the Laplacian are determined respectively as solutions of

$$
J_{M}(\sqrt{\lambda} r)=0, \text { and } J_{M}^{\prime}(\sqrt{\lambda} r)=0
$$

We cannot solve these explicitly to find the eigenvalues. However, the flat disk is related by a Weyl transformation to the stereographic projection onto the equatorial plane of the hemisphere of radius $r$ with the standard polar metric.

The eigenfunctions on the hemisphere are the spherical harmonics with $l+m$ odd for Dirichlet and $l+m$ even for Neumann boundary conditions. The 
corresponding eigenvalues are

$$
\lambda_{l}=l(l+1) / r^{2} \text { with degeneracy }=\left\{\begin{array}{c}
l, \text { Dirichlet } \\
l+1, \text { Neumann }
\end{array}\right.
$$

The determinants are defined as

$$
\operatorname{det}_{\left(\begin{array}{c}
D \\
N
\end{array}\right)}^{\prime} \Delta=\exp \left[-2 \ln (r) \zeta_{\left(\begin{array}{c}
D \\
N
\end{array}\right)}(0)-\zeta_{\left(\begin{array}{l}
D \\
N
\end{array}\right)}^{\prime}(0)\right]
$$

where

$$
\zeta_{\left(\begin{array}{l}
D \\
N
\end{array}\right)}(s)=\sum_{1}^{\infty}\left(\begin{array}{c}
l \\
l+1
\end{array}\right)[l(l+1)]^{-s} .
$$

For the zeta function prime denotes differentiation.

These zeta functions can be evaluated by the method used to calculate the determinant for the sphere in App. C of ref. [3]. The results are

$$
\begin{aligned}
& \zeta_{D}(0)=1 / 6, \quad \zeta_{N}(0)=-5 / 6, \\
& \zeta_{D}^{\prime}(0)=2 \zeta_{R}^{\prime}(-1)+(1 / 2) \ln (2 \pi)-1 / 4, \\
& \zeta_{N}^{\prime}(0)=2 \zeta_{R}^{\prime}(-1)-(1 / 2) \ln (2 \pi)-1 / 4,
\end{aligned}
$$

where $\zeta_{R}(s)$ is the standard Riemann zeta function. Take the flat metric on the disk as the gauge choice. Then

$$
\hat{K}=0, \quad \hat{\kappa}_{g}=1 / r .
$$

The metric for the hemisphere projected onto the disk is

$$
d s^{2}=4 d z d z /\left[1+|z|^{2} / r^{2}\right]^{2} .
$$

The gauge parameter on the hemisphere is

with

$$
\phi=\ln \left(2 /\left[1+|z|^{2} / r^{2}\right]\right)
$$

For the hemisphere

$$
\left.\phi\right|_{\partial M}=0 \text {. }
$$

$$
L\left(\phi, \delta_{a b}\right)=\int_{|z|<r} d z d \bar{z}|z|^{2} /\left[r^{2}+|z|^{2}\right]^{2}=2 \pi(\ln 2-1 / 2) .
$$

The invariants of Eqs. $(4,5)$ enable us to express the determinants on the flat disk as

$$
\begin{aligned}
& \operatorname{det}_{D} \Delta=2^{-1 / 6} \pi^{-1 / 2} r^{-1 / 3} \exp \left[-2 \zeta_{R}^{\prime}(-1)-5 / 12\right] \\
& \operatorname{det}_{N}^{\prime} \Delta=2^{-1 / 6} \pi^{1 / 2} r^{5 / 3} \exp \left[-2 \zeta_{R}^{\prime}(-1)+7 / 12\right] .
\end{aligned}
$$

B. Flat Annulus of Radii $r_{1}<r_{2}$. The flat annulus is related by Weyl scaling to a cylinder of length $l$ and unit circumference with a flat metric. The cylinder can be represented in the complex $w$-plane by a rectangle of unit width and length $l$ with the sides at $\operatorname{Re} w=0$ and $\operatorname{Re} w=1$ identified. The cylinder is mapped onto the annulus in the $z$-plane by

with

$$
z=r_{2} \exp (2 \pi i w)
$$

$$
2 \pi l=\ln \left(r_{2} / r_{1}\right) .
$$


Take as the gauge the metric of the cylinder

$$
d s^{2}=d z d \bar{z} /(2 \pi|z|)^{2} .
$$

Then for the flat annulus

$$
\phi=\ln (2 \pi|z|)
$$

and

$$
L\left(\phi, \hat{g}_{a b}\right)=\int_{r_{1}<|z|<r_{2}} d z d \bar{z} /\left(4|z|^{2}\right)=\pi \ln \left(r_{2} / r_{1}\right) .
$$

For the cylinder the eigenvalues of $\Delta$ are

with

$$
\lambda_{m, n}=(2 \pi m)^{2}+(\pi n / l)^{2}
$$

and

$$
m \in \mathbb{Z}
$$

$$
\begin{aligned}
& n \geqq 1, \text { Dirichlet } \\
& n \geqq 0 \text {, Neumann. }
\end{aligned}
$$

The evaluation of the zeta-function regulated determinants is essentially the same as the calculation for the torus in ref. [7], and gives

$$
\operatorname{det}_{D} \Delta=\operatorname{det}_{N}^{\prime} \Delta=2 l \exp [-\pi l / 3][f(\exp [-4 \pi l])]^{-2},
$$

where $f$ is the partition function

$$
f(x)=\prod_{1}^{\infty}\left(1-x^{n}\right)^{-1}
$$

Equating the invariants for the flat annulus and the cylinder gives the determinants for the annulus,

$$
\begin{aligned}
& \operatorname{det}_{D} \Delta=(1 / \pi)\left(r_{1} / r_{2}\right)^{1 / 3} \ln \left(r_{2} / r_{1}\right)\left[f\left(\left[r_{1} / r_{2}\right]^{2}\right)\right]^{-2}, \\
& \operatorname{det}_{N}^{\prime} \Delta=2 \pi\left(r_{1} / r_{2}\right)^{1 / 3}\left[\left(r_{2}\right)^{2}-\left(r_{1}\right)^{2}\right]\left[f\left(\left[r_{1} / r_{2}\right]^{2}\right)\right]^{-2} .
\end{aligned}
$$

Acknowledgements. The author thanks Dr. M. Rocek and Mr. C. Varughese for helpful discussions. This work was supported in part by the National Science Foundation under Grant PHY-85-07627.

\section{References}

1. Polyakov, A. M.: Quantum geometry of bosonic strings. Phys. Lett. 103B, 211-213 (1981)

2. Alvarez, O.: Theory of strings with boundaries: Fluctuations, topology and quantum geometry. Nucl. Phys. B216, 125-184 (1983)

3. Weisberger, W. I.: Normalization of the path integral measure and the coupling constants for bosonic strings. Nucl. Phys. B284, 171-200 (1987)

4. Hawking, S.: Zeta function regularization of path integrals in curved spacetime. Commun. Math. Phys. 55, 133-148 (1977)

5. McKean, H. P., Singer, I. M.: Curvature and the eigenvalues of the Laplacian. J. Differ. Geom. 1, 43-69 (1967)

6. Balian, R., Bloch, C.: Distribution of the eigenfrequencies for the wave equation in a finite domain. II. Electromagnetic field. Riemannian spaces. Ann. Phys. 64, 271-307 (1971)

7. Polchinski, J.: Evaluation of the one-loop string path integral. Commun. Math. Phys. 104, 37-47 (1986)

Communicated by A. Jaffe

Received March 18, 1987 